\title{
Robust Bulk Superconductivity by Giant Proximity Effect in Weyl Semimetal-superconducting $\mathrm{NbP} / \mathrm{NbSe} 2$ Composites
}

\section{Yejin Lee}

Kyung Hee University

Omkaram Inturu

Kyung Hee University

Jin Hee Kim

Kyung Hee University

Jong-Soo Rhyee ( $\nabla$ jsrhyee@khu.ac.kr)

Kyung Hee University

\section{Research Article}

Keywords: NbP, NbSe2, Superconductivity, bulk composite, Weyl semimetal

Posted Date: September 21st, 2021

DOI: https://doi.org/10.21203/rs.3.rs-890116/v1

License: (a) (i) This work is licensed under a Creative Commons Attribution 4.0 International License. Read Full License 


\title{
Robust bulk superconductivity by giant proximity effect in Weyl semimetal-superconducting $\mathrm{NbP} / \mathrm{NbSe}_{2}$ composites
}

\author{
Yejin Lee ${ }^{\dagger}$, Omkaram Inturu, Jin Hee Kim, Jong-Soo Rhyee* \\ Department of Applied Physics and Integrated Education Program for Frontier Science and \\ Technology (BK21 Four), Institute of Natural Sciences, Kyung Hee University, Yong-In 17104, \\ Republic of Korea
}

\begin{abstract}
We synthesize Weyl semimetal/superconductor $\mathrm{NbP} / \mathrm{NbSe}_{2}$ composite and observe stable bulk superconductivity at $T_{c}=7.2 \mathrm{~K}, 6.9 \mathrm{~K}$, and $6.8 \mathrm{~K}$ for $\mathrm{NbSe}_{2}$ crystal, $\mathrm{NbP} / \mathrm{NbSe}_{2}(1: 1)$, and $\mathrm{NbP} / \mathrm{NbSe}_{2}$ (2:1) composites, respectively, despite large volume fraction of non-superconducting $\mathrm{NbP}$ phase. From the Ginzburg-Landau theory, the $H_{c 2}(0)$ is significantly enhanced in $\mathrm{NbP} / \mathrm{NbSe}_{2}$ composites [22 $\mathrm{T}(1: 1)$ and $18.5 \mathrm{~T}(2: 1)$ ] comparing with the pristine $\mathrm{NbSe}_{2}$ crystal $(8 \mathrm{~T})$. The bulk superconductivity in Weyl semimetal/superconductor composite cannot be simply described by the de Gennes-Meissner theory in a proximity effect. From the electrical transport, magnetization, and heat capacity measurement, we obtain various superconducting parameters. The superconducting properties indicate that the $\mathrm{NbP} / \mathrm{NbSe}_{2}$ composite is far from the conventional BCS superconductivity. It suggests that the Weyl semimetal/superconductor composite can have giant proximity effect, resulting in the stable bulk superconductivity in a composite with sizable volume fraction of non-superconducting Weyl semimetals. The giant proximity effect in Weyl semimetal/superconductor interface can have a platform to investigate the proximity induced Weyl semimetallic superconducting states.
\end{abstract}

KEYWORDS : NbP, NbSe, Superconductivity, bulk composite, Weyl semimetal.

${ }^{\dagger}$ Present address : Helmholtzstraße 20, IFW-Dresden, 01069 Dresden, Germany

${ }^{*}$ Correspondence should be addressed to J.S.R. (jsrhyee@khu.ac.kr ) 


\section{Introduction}

Since the discovery of topological insulator, the search on the various topological insulators such as topological crystalline insulator ${ }^{1}$, topological Anderson insulator ${ }^{2,3}$, topological Mott insulator ${ }^{4}$, etc. have been sought out for new state of matter in condensed matter physics. The topological materials have unusual physical properties resulting from massless Dirac fermion, which can control the fascinating transport properties. On the other hand, the detection of surface properties is limited by using the bulk transport measurements because surface to volume ratio is negligible in bulk compounds. Instead, Dirac and Weyl semimetals show topologically protected states in bulk transport properties. A Weyl semimetal is driven by the symmetry breaking either time reversal symmetry or inversion symmetry from Dirac semimetal ${ }^{5}$. Weyl semimetals have a band structure with two Weyl points as one pair, which makes Fermi arcs on a Fermi surface. Weyl points keep opposite chirality and can be either a source or a sink in the form of topological charge, which can be viewed as a monopole. In particular, the bulk properties of Weyl semimetals with the exotic features like chiral anomaly ${ }^{5-7}$, ultra high carrier mobility ${ }^{8}$, and large magnetoresistance ${ }^{9}$ are unconventional anomalous transport behavior in terms of robust band topology.

Superconductivity in Weyl semimetals is of great interest but there are not many investigations on the coexistence of bulk superconductivity and a type-I Weyl semimetal (WSM). Type-I Weyl semimetal $\mathrm{TaP}$ becomes superconductor under high pressure above $100 \mathrm{GPa}$, accompanying structural phase transition at $70 \mathrm{GPa}^{10}$. On the other hand, there have been several reports on the coexistence of superconductivity in type-II Weyl semimetals such as $\mathrm{MoTe}_{2}$ and $\mathrm{WTe}_{2}{ }^{11-14}$. Nevertheless, there are many theoretical suggestions on the existence of Weyl superconductors ${ }^{15-17}$. According to the theoretical prediction, WSM can be a superconducting phase caused by superconducting proximity in heterostructure consisting of a WSM and a superconductor ${ }^{18}$. Experimentally, ion irradiation in NbAs induces breaking the $\mathrm{Nb}$ and As bond which leads the natural enrichment of $\mathrm{Nb}$ at the surface. Because the excessive $\mathrm{Nb}$ becomes superconducting state at $T_{c} \sim 3.5$ $\mathrm{K}$, the surface proximity effect makes a superconducting state in Weyl semimetal ${ }^{19}$.

Niobium phosphorus (NbP) which has a noncentrosymmetric structure $\left(\mathrm{I} 4{ }_{1} \mathrm{md}\right)$ is a representative Weyl semimetal20-25. There are 12 Weyl pairs of Weyl nodes in bulk Brillouin zone of $\mathrm{NbP}$ based on the first principle calculation ${ }^{26,27}$. It has been shown that the NbP has an ultra-high carrier mobility and chiral anomaly-induced negative magnetoresistance like as typical features of WSM $^{24,28}$.

As a good candidate superconducting material for a heterostructure with $\mathrm{NbP}$, the $\mathrm{NbSe}_{2}$ exhibits the superconducting transition at $T_{c}=7.2 \mathrm{~K}$ with coexistence of charge density wave at $T_{C D W}=33 \mathrm{~K}$. 
It has the similar lattice parameters $(a=3.44$ and $\mathrm{c}=12.54)$ with that of $\mathrm{NbP}(\mathrm{a}=3.3324$ and $c=11.3705$ ), which anticipates the coherent interface between $\mathrm{NbP}$ and $\mathrm{NbSe}_{2}$ due to lattice match. Superconductivity also can be induced via the proximity effect, through the interface diffusion of Cooper pairs. $\mathrm{NbSe}_{2}$ has been known as a Ising type superconductivity ${ }^{29,30}$. Ising superconductivity has the anomalously large in-plane critical magnetic field ${ }^{31}$. The high spin-orbit coupling (SOC) with inversion symmetry breaking locks the pseudospins near $\mathrm{K}$ and $\mathrm{K}^{\prime}$ points which are parallel to the caxis. By the time-reversal symmetry, the pseudospins at $\mathrm{K}$ and $\mathrm{K}^{\prime}$ are formed as antiparallel direction with degenerated energy. This unconventional paring of pseudospins can survive under exceedingly high in-plane magnetic fields comparing with the Pauli limit $32,33$.

Here, we investigated the superconducting properties in $\mathrm{NbP} / \mathrm{NbSe}_{2}$ bulk composite, to explore the proximity induced superconductivity on the $\mathrm{NbP}$, anticipating the coexistence of Weyl semimetallic property as well as the superconducting properties. The magnetic, electronic transport, and heat capacity properties show the robust type-II superconductivity even large volume fraction of non-superconducting $\mathrm{NbP}\left(\mathrm{NbP} / \mathrm{NbSe}_{2}=2: 1\right.$ in molar ratios). In addition, we observed the enhancement of the upper critical field $\mathrm{H}_{\mathrm{c} 2}$ and the reduction of coherence length $\xi$ in the composite.

\section{Results}

Fig. 1a shows the X-ray diffraction patterns of the crystalline $\mathrm{NbSe}_{2}$ (purple) and $\mathrm{NbP} / \mathrm{NbSe}_{2}$ composites with different molar concentrations of $\mathrm{NbP} / \mathrm{NbSe}_{2}=1: 1$ (red) and 2:1 (black). The reference peaks are also shown for comparison. All labeled diffraction peaks of the grown single crystal of $\mathrm{NbSe}_{2}$ are aligned along the (001) peaks, which is indexed by the hexagonal crystalline structure. The diffraction peaks of the composites 1:1 and 2:1 coincide with the standard Bragg peaks with coexisting phases of $\mathrm{NbP}$ [PDF\#17-0882] and $\mathrm{NbSe}_{2}$ [PDF\#18-0921], indicating the mixed phase composite not a doping. The lattice parameters of NbP phase in the composites are $\mathrm{a}=3.332 \AA, \mathrm{c}=$ 11.638 $\AA, \mathrm{V}=126.19 \AA^{3}\left[\mathrm{NbP} / \mathrm{NbSe}_{2}(1: 1)\right]$ and $\mathrm{a}=3.326 \AA, \mathrm{c}=11.408 \AA, \mathrm{V}=126.19 \AA^{3}\left[\mathrm{NbP} / \mathrm{NbSe}_{2}\right.$ (2:1)]. The lattice volume of the NbP is not changed for different molar ratio concentrations of $\mathrm{NbP} / \mathrm{NbSe}_{2}$ composites. The lattice parameters of the $2 \mathrm{H}-\mathrm{NbSe}_{2}$ phase on the composites are $\mathrm{a}=$ $3.450 \AA, c=12.530 \AA, V=128.36 \AA^{3}\left[\mathrm{NbP} / \mathrm{NbSe}_{2}(1: 1)\right]$ and $\mathrm{a}=3.443 \AA, \mathrm{c}=12.550 \AA, \mathrm{V}=128.85 \AA^{3}$ $\left[\mathrm{NbP} / \mathrm{NbSe}_{2}(2: 1)\right]$, respectively. The lattice volume of hexagonal $2 \mathrm{H}-\mathrm{NbSe}_{2}$ little bit increased by increasing the c-axis lattice parameter for increasing NbP concentration, but it is close to the lattice parameters of single crystalline $\mathrm{NbSe}_{2}(\mathrm{a}=3.45 \AA$ and $\mathrm{c}=12.544 \AA$ ). It shows the stable phase mixing of $\mathrm{NbP}$ in $\mathrm{NbSe}_{2}$ matrix. 
The morphologies of the composites show the hexagonal crystal structure of $\mathrm{NbSe}_{2}$ with the agglomerated NbP particles in Fig. $1 \mathrm{~b}\left[\mathrm{NbP} / \mathrm{NbSe}_{2}(1: 1)\right]$ and 1c (2:1), respectively. The typical sizes of crystalline $\mathrm{NbSe}_{2}$ show tens of $\mu \mathrm{m}$ scale, while the grain size of $\mathrm{NbP}$ have wide range of particle sizes under several $\mu \mathrm{m}$ scale.
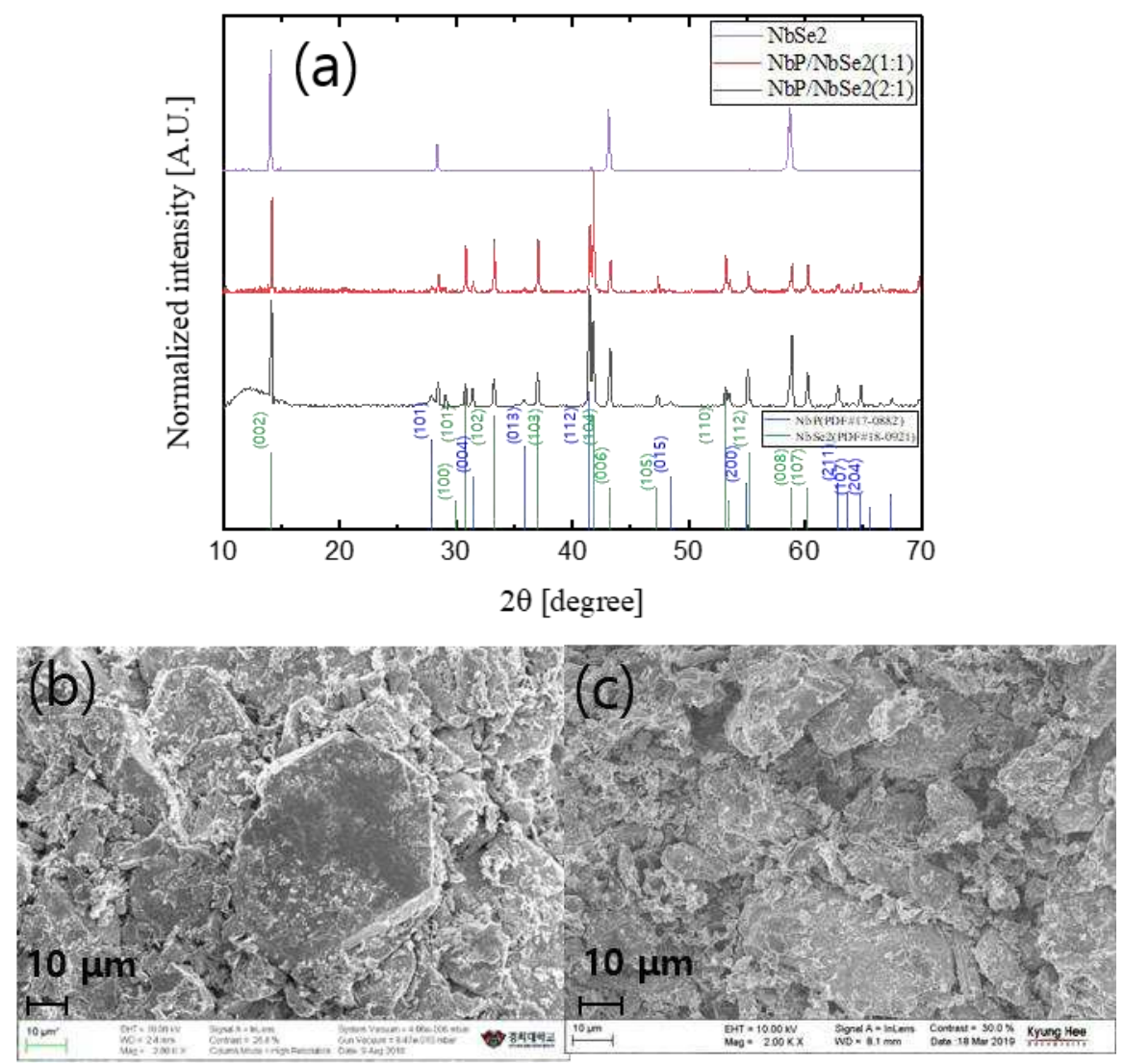

Figure 1. X-ray diffraction (XRD) patterns and scanning electron microscope (SEM) images. XRD patterns for the $\mathrm{NbSe}_{2}$ single crystal (purple), $\mathrm{NbP} / \mathrm{NbSe}_{2}$ composites with molar ratios of 1:1 (red) and 2:1 (black) composites (a). Scanning electron microscope (SEM) images of $\mathrm{NbP} / \mathrm{NbSe}_{2}$ composites with molar ratios of 1:1 (b) and 2:1 (c) molar ratios.

The phase separation of $\mathrm{NbP}$ and $\mathrm{NbSe}_{2}$ can be clearly seen in the elemental mapping from the energy dispersive X-ray spectroscopy (EDX), as presented in Fig. 2. Fig. 2a-d and 2e-h present the elemental mapping on the $\mathrm{NbP} / \mathrm{NbSe}_{2}(1: 1)$ and (2:1) composites, respectively. While the $\mathrm{Nb}$ is widely distributed as shown in Fig. $2 \mathrm{~b}$ and $2 \mathrm{f}$, the $\mathrm{P}$ and Se elements are clearly distinguishable. For example, Fig. 2a shows clear separation of Se (red dots) and P (green dots) in $\mathrm{NbP} / \mathrm{NbSe}_{2}$ (1:1). Fig. 2e is the EDX mapping of the $\mathrm{NbP} / \mathrm{NbSe}_{2}(2: 1)$ composite, showing clear phase separation of Se (pink 
dots) and P (bright green dots). Through quantitative analysis of EDX, the concentration of phosphorus in 2:1 sample is larger than those in 1:1 composite.

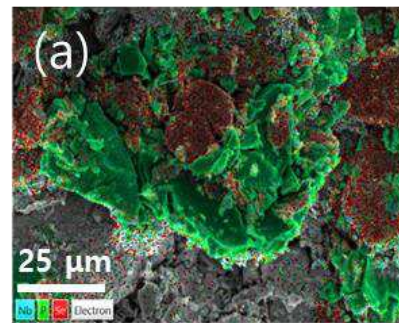

(e)
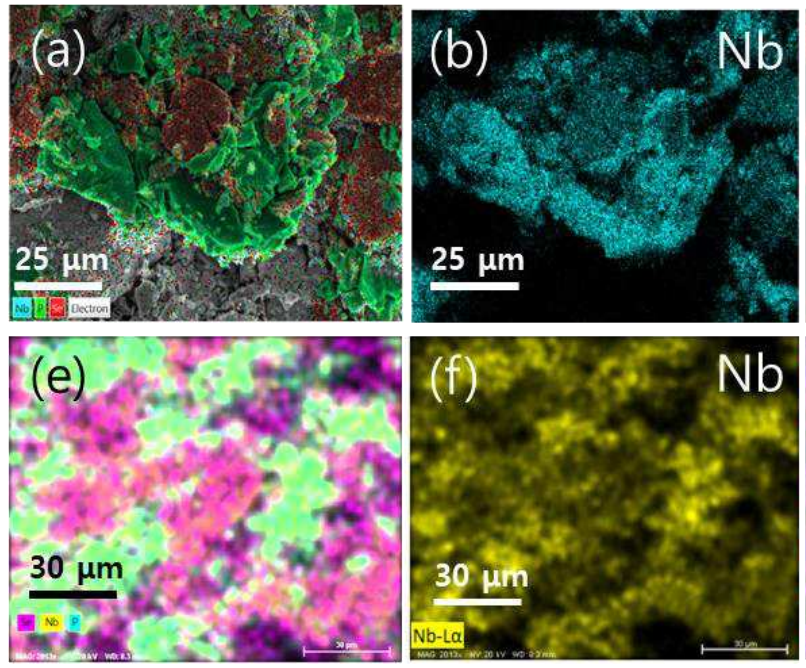
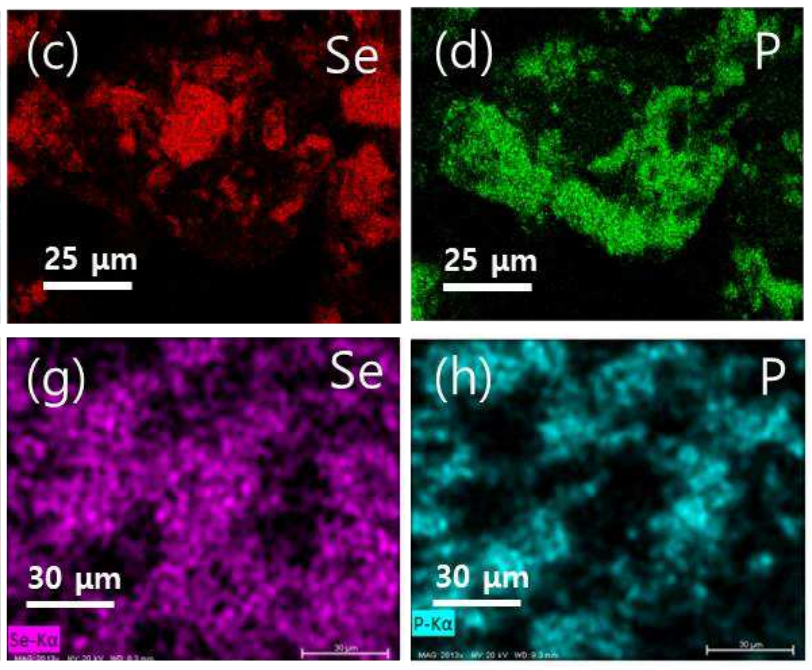

Figure 2. Elemental mapping from the energy dispersive $X$-ray spectroscopy (EDX) of the $\mathrm{NbP} / \mathrm{NbSe}_{2}(1: 1)(\mathrm{a}-\mathrm{d})$ and $\mathrm{NbP} / \mathrm{NbSe}_{2}(2: 1)(\mathrm{e}-\mathrm{h})$ composites.
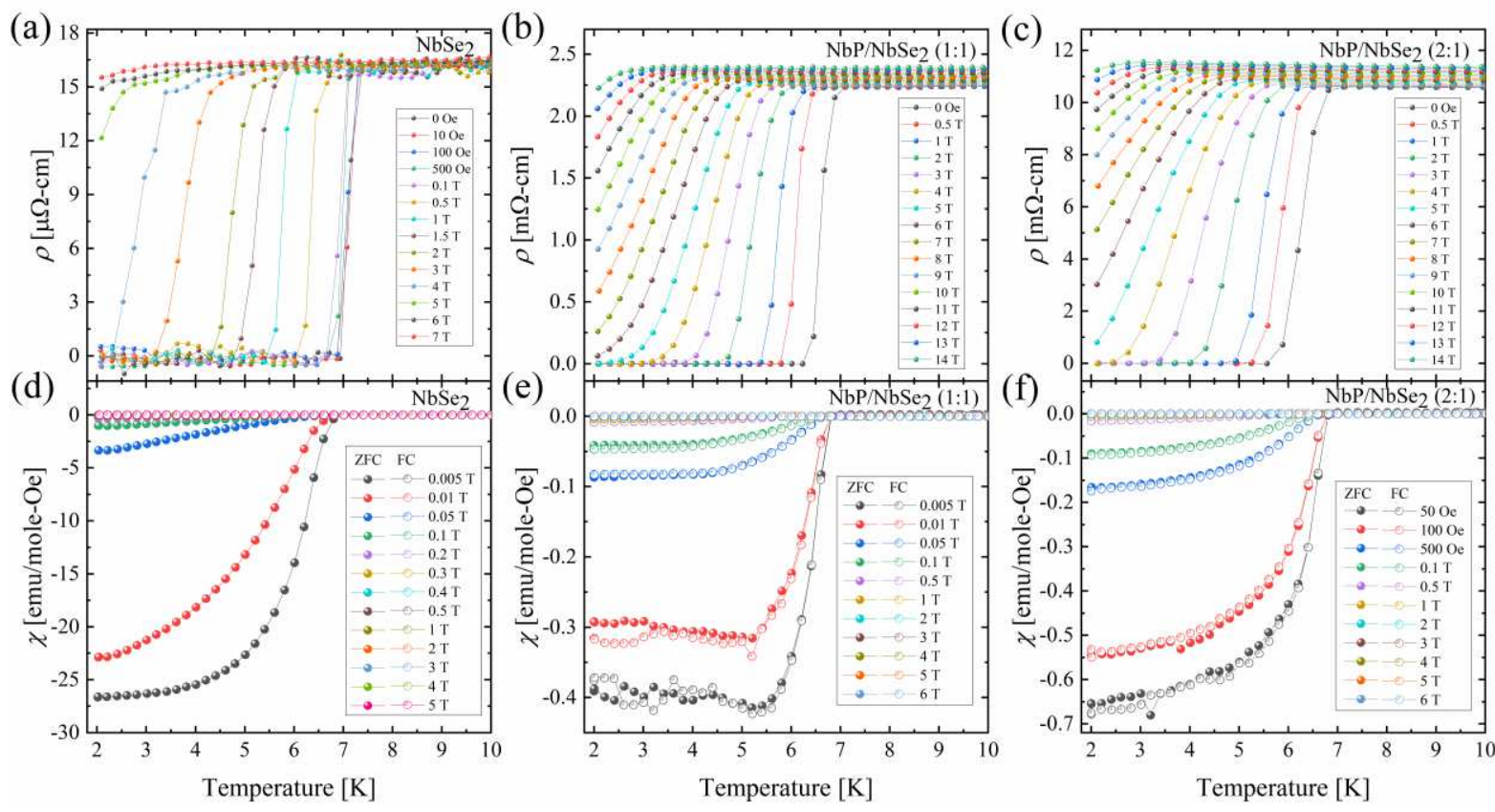

Figure 3. Temperature-dependent electrical resistivity $\boldsymbol{\rho}(\boldsymbol{T})$ of the $\mathrm{NbSe}_{2}$ crystal (a), $\mathrm{NbP} / \mathrm{NbSe}{ }_{2}$ $(1: 1)$ (b), and $\mathrm{NbP} / \mathrm{NbSe}_{2}(2: 1)$ (c). Temperature-dependent magnetic susceptibility $\chi(T)$ of the $\mathrm{NbSe}_{2}$ crystal (d), $\mathrm{NbP} / \mathrm{NbSe}_{2}$ (1:1) (e), and $\mathrm{NbP} / \mathrm{NbSe}_{2}$ (2:1) (f). 
Fig.s 3 represents the temperature-dependent electrical resistivity $\rho(T)$ 3a-c and magnetization $M(T) 3 d$-f from 2 to $10 \mathrm{~K}$ under various applied magnetic fields for the pristine $\mathrm{NbSe}_{2} 3 \mathrm{a}$ and $3 \mathrm{~d}$, $\mathrm{NbP} / \mathrm{NbSe}_{2}(1: 1) 3 b$ and $3 \mathrm{e}$, and $\mathrm{NbP} / \mathrm{NbSe}_{2}$ (2:1) 3c and 3f respectively. It is noteworthy that the superconducting transitions are observed in the $\mathrm{NbP} / \mathrm{NbSe}_{2}$ composites even large volume fraction of $\mathrm{NbP}$ (2:1). The onset critical temperatures of $\mathrm{NbSe}_{2}, \mathrm{NbP} / \mathrm{NbSe}_{2}$ (1:1), and $\mathrm{NbP} / \mathrm{NbSe}_{2}$ (2:1) composites are $T_{c}=7.2 \mathrm{~K}, 6.9 \mathrm{~K}$, and $6.8 \mathrm{~K}$, respectively. The $T_{c}(7.2 \mathrm{~K})$ of $\mathrm{NbSe}_{2}$ is same with the previously reported one ${ }^{34}$. It is surprising because the non-superconducting NbP composite with a sizable phase separation $(10 \sim 50 \mu \mathrm{m})$ in superconducting $\mathrm{NbSe}_{2}$ compound show stable superconducting transition. Even though the transition widths of superconducting transition $\Delta T_{c}=$ $T_{c}^{\text {onset }}-T_{c}^{\text {zero }}$ are increased with increasing $\mathrm{NbP}$ mole fraction, the transition widths $\Delta T_{c}$ of $\mathrm{NbP} / \mathrm{NbSe}_{2}$ composites are very sharp comparable with the pristine $\mathrm{NbSe}_{2}$ crystal; $\Delta T_{c}=0.2 \mathrm{~K}$ $\left(\mathrm{NbSe}_{2}\right), 0.6 \mathrm{~K}$ (1:1 composite), and $1.2 \mathrm{~K}$ (2:1 composite) without magnetic fields.

Fig.s $3 \mathrm{~d}-\mathrm{f}$ shows the magnetic susceptibility as a function of temperature $\chi(T)$ measured in zerofield cooled (ZFC, closed symbols) and field-cooled (FC, open symbols) cycles under various static magnetic fields for the $\mathrm{NbSe}_{2}$ and the $\mathrm{NbP} / \mathrm{NbSe}_{2}$ composites (1:1 and 2:1). The onset temperature of the diamagnetic signal is close to $T_{c}$ defined in resistivity measurement. The magnetic susceptibilities of the $\mathrm{NbP} / \mathrm{NbSe}_{2}$ composites are weaker than the $\chi(T)$ value of the $\mathrm{NbSe}_{2}$ due to the presence of non-magnetic NbP. When we estimate the magnetic susceptibility in the composites, we used the composite density by $\rho=\left[\rho_{1} V_{1}+\rho_{2} V_{2}\right] / V$, where $\rho_{1(2)}$ and $V_{1(2)}$ are the density and volume of the $\mathrm{NbP}\left(\mathrm{NbSe}_{2}\right)$ and $V$ is the total volume of the sample. Conventional type-II superconductors show strong hysteric behavior of FC and ZFC measurements by strong flux pinning. It is very noteworthy that the $\chi(T)$ of the $\mathrm{NbP} / \mathrm{NbSe}_{2}$ composites displays strong diamagnetic signal for the FC sequence, which is not significantly different with the ZFC measurement. This strong diamagnetic signal in FC measurement is also exceptional in type II superconductors. The FC and ZFC measurement of $\mathrm{NbSe}_{2}$ show the paramagnetic signal in FC measurement with strong diamagnetic signal in ZFC measurement, which is conventional behavior. This small differences between $\mathrm{ZFC}$ and $\mathrm{FC}$ curves in the $\mathrm{NbP} / \mathrm{NbSe}_{2}$ composites indicate a weak flux pinning but strong superconducting state in the composites.

\section{Discussion}

To be a bulk superconductivity in a composite, the Cooper pair should not be scattered near the superconducting/normal metal interface. The only reasonable description on the bulk 
superconductivity in the $\mathrm{NbP} / \mathrm{NbSe}_{2}$ composite is the proximity effect between superconducting and normal interface.

From the De Gennes and Meissner theory, the cooper pair electrons in superconductor can penetrate into a normal metal as a characteristic length scale $\xi_{n}$ where it is a coherent length of normal metal as a following relation ${ }^{35,36}$ :

$$
I_{c} \approx \frac{\pi}{2 e R_{n}} \frac{\Delta_{i}^{2}}{k T_{c}} \frac{d}{\xi_{n}} e^{-d / \xi_{n}}
$$

where $R_{n}$ is the resistance of the junction in its normal state, $\Delta_{i}$ is the superconducting energy gap at normal interface, $T_{c}$ is the superconducting transition temperature of superconducting side, $d$ is the thickness of normal layer. In clean limit $\left(\xi_{s} \ll l_{n}\right)$, the characteristic length in normal metal $\xi_{n, c}$ is presented by $\xi_{n, c}=\hbar v_{n} / 2 \pi k T$, where $v_{n}$ is the Fermi velocity of normal metal. In dirty limit $\left(l_{n} \ll\right.$ $\xi_{s}$ ), the characteristic length is given by $\xi_{n, d}=\sqrt{\hbar v_{n} l_{n} / 6 \pi k T}$, where $l_{n}$ is the mean free path of normal metal and $\xi_{s}$ is the coherence length of superconductor.

Many high temperature superconductors showed that the characteristic length in normal metal by proximity effect is less than $10 \AA$. For example, the $\mathrm{YBa}_{2} \mathrm{Cu}_{3} \mathrm{O}_{7}\left(T_{c}=92 \mathrm{~K}\right)$ and Co-doped $\mathrm{PrBa}_{2} \mathrm{Cu}_{3} \mathrm{O}_{7}$ interface (normal metal) interface exhibits the $\xi_{n}=6 \AA .{ }^{37}$ For $\xi_{n}=5 \mu \mathrm{m}$ in $\mathrm{NbP} / \mathrm{NbSe}_{2}(2: 1)$ composite, which is necessary for bulk superconductivity in the composite, the Fermi velocity of the normal metal should be $2.46 \times 10^{7} \mathrm{~m} / \mathrm{s}$ in a clean limit. The Fermi velocities of the conventional metallic materials are $1.39 \times 10^{6} \mathrm{~m} / \mathrm{s}(\mathrm{Ag}), 1.52 \times 10^{6} \mathrm{~m} / \mathrm{s}(\mathrm{Cu})$, and $2.03 \times 10^{6} \mathrm{~m} / \mathrm{s}(\mathrm{Al})$, respectively ${ }^{38}$. The Fermi velocity of NbP is estimated by $3 \times 10^{5} \mathrm{~m} / \mathrm{s}$, which is much lower than the expected value in terms of the de Gennes-Meissner criteria $^{39}$. On the other hand, it was observed the giant proximity effect (GPE) in high $\mathrm{T}_{\mathrm{c}}$ cuprate superconductors ${ }^{40}$. The supercurrents can persist in Josephson junctions with high $\mathrm{T}_{\mathrm{c}}$ cuprates as thick as $100 \mathrm{~nm} \sim 1 \mu \mathrm{m}^{41-46}$. The de Gennes-Meissner criteria is based on the junctions between conventional s-wave metallic BCS (Bardeen-Cooper-Schrieffer) superconductor and metals. The composites or interfaces comprised with d-wave superconductivity or Weyl semimetals do not follow the de Gennes-Meissner equation. Therefore, the robust bulk superconductivity in $\mathrm{NbP} / \mathrm{NbSe}_{2}$ composite may come from the giant proximity effect in Weyl semimetallic/superconducting bulk composite.

The lower critical field $H_{c 1}$ can be observed in the magnetic field dependent electrical resistivity measurements $\rho(H)$, as presented in Fig. 4. The $H_{c 1}$ at $2 \mathrm{~K}$ of $\mathrm{NbSe}_{2}$ crystal shows $4.4 \mathrm{~T}$ and the $H_{c 1}$ are increased in the $\mathrm{NbP} / \mathrm{NbSe}_{2}$ composites by about $6 \mathrm{~T}$. The $H_{c 1}$ is decreased with increasing temperatures such as $3.2 \mathrm{~T}(3 \mathrm{~K}), 2.4 \mathrm{~T}(4 \mathrm{~K})$, and $1.5 \mathrm{~T}(5 \mathrm{~K})$ in $\mathrm{NbSe}_{2}$ crystal. The decreases of $H_{c 1}$ in the composites are similar to the $\mathrm{NbSe}_{2}$ crystal; for example, $4 \mathrm{~T}(3 \mathrm{~K}), 2.8 \mathrm{~T}(4 \mathrm{~K})$, and $1.5 \mathrm{~T}(5 \mathrm{~K})$. On 
the other hand, the transition rates of critical field $H_{c 1}$ in the composites are broader than the one of $\mathrm{NbSe}_{2}$ crystal.
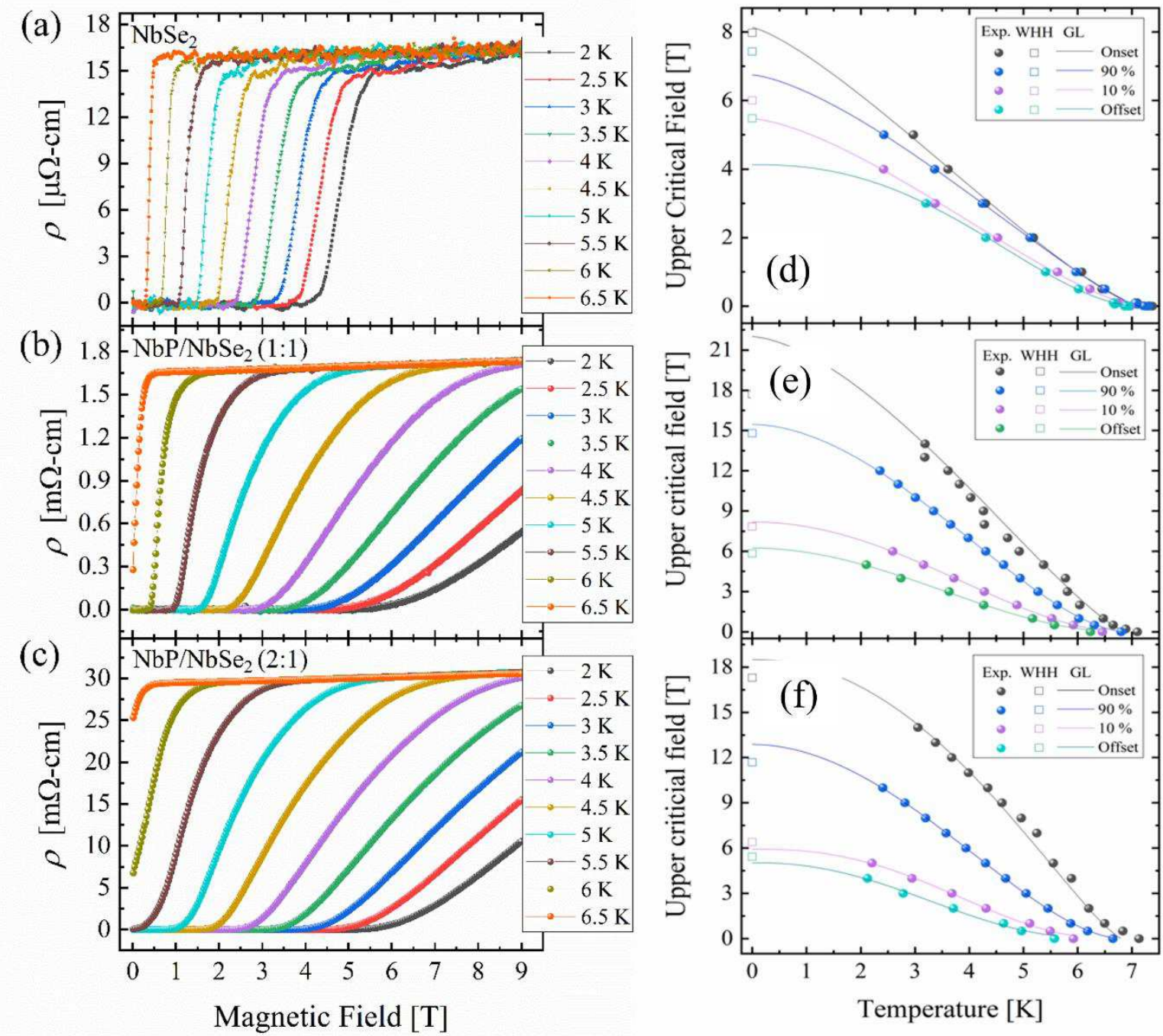

Figure 4. Magnetic field dependent isothermal electrical resistivity $\rho(H)$ at different temperatures and Temperature-dependent upper critical field $H_{c 2}(T) . \rho(H)$ for the $\mathrm{NbSe}_{2}$ crystal (a), $\mathrm{NbP} / \mathrm{NbSe}_{2}(1: 1)$ (b), and $\mathrm{NbP} / \mathrm{NbSe}_{2}$ (2:1) (c) composites. Temperature-dependent upper critical field $H_{c 2}(T)$ of the $\mathrm{NbSe}_{2}$ crystal (d), $\mathrm{NbP} / \mathrm{NbSe}_{2}$ (1:1) (e), and $\mathrm{NbP} / \mathrm{NbSe}_{2}$ (2:1) (f) composites. Closed symbols are experimental values from the electrical resistivity and open symbols are $H_{c 2}(0)$ obtained by the WHH formula. Lines are the fitting from the Ginzburg-Landau theory. 
From the temperature- and field-dependent electrical resistivity and magnetic susceptibility measurements, we can estimate the superconducting parameters such as the upper critical field $H_{c 2}$, coherence length $\xi$, Ginzburg-Landau parameter $\kappa$, and penetration depth $\lambda$. The upper critical fields $H_{c 2}(T)$ with temperature are plotted in Fig. 4d-f. Black, blue, purple, and sky-blue symbols represent the $H_{c 2}(T)$ values obtained from the normal-superconducting onset, $90 \%$ and $10 \%$ points from the onset, and zero-resistivity offset points, respectively. Lines are theoretical fitting with the GingburgLandau (GL) equation $H_{c 2}(T)=H_{c 2}(0)\left[1-\left(T / T_{c}\right)\right]^{2}$, which is well fitted with the experimental data. The zero-temperature limit of upper critical field $H_{c 2}(0)$ can be estimated by the GL equation. For example, the $H_{c 2}(0)$ s of the samples are $8 \mathrm{~T}$ for $\mathrm{NbSe}_{2}, 22 \mathrm{~T}$ for $\mathrm{NbP} / \mathrm{NbSe}_{2}(1: 1)$, and $18.5 \mathrm{~T}$ for $\mathrm{NbP} / \mathrm{NbSe}_{2}$ (2:1) composites, respectively, from the onset point criteria. It is known that the $\mathrm{NbSe}_{2}$ is an Ising type superconductivity with strong anisotropic $\mathrm{H}_{c 2}(0)^{47}$.

The low temperature upper critical field of the $\mathrm{NbSe}_{2}$ showed $14 \mathrm{~T}$ for along the in-plane and 4 $\mathrm{T}$ for the out-of-plane direction ${ }^{48}$. Also, other reports presented the $H_{c 2,} / /(0)=8 \mathrm{~T}$ and $H_{c 2},{ }^{\perp}(0)=2 \mathrm{~T}$ for in-plane and out-of-plane direction, respectively ${ }^{49}$. It indicates that the $H_{c 2}(0)$ depends on the sample conditions and $H_{c 2}(0)=8 \mathrm{~T}$ is the reasonable value along the in-plane upper critical field. It is noteworthy that the non-magnetic $\mathrm{NbP}$ composites $\mathrm{NbP} / \mathrm{NbSe}_{2}$ show exceeding value of the $H_{c 2}(0)=$ $14 \mathrm{~T}$ for the in-plane direction of $\mathrm{NbSe}_{2}$ even in polycrystal and composite samples. It is very exceptional because non-superconducting composite should degrade the superconductivity.

The $H_{c 2}(0)$ can also be obtained from the Werthamer-Helfand-Hohenberg (WHH) formula, given by $H_{c 2}(0)=-\left.0.693 T_{c}\left(\mathrm{~d} H_{c 2} / \mathrm{d} T\right)\right|_{T=T c} 48,50$. The $H_{c 2}(0)$ from the WHH formula is little bit different from the values obtained by the GL theory. Table 1 presents the comparison of the $H_{c 2}(0)$ values obtained from the GL theory and WHH formula. While the $H_{c 2}(0)$ values from the onset points by WHH formula are little bit lower than the values by the GL theory, the $H_{c 2}(0)$ values from the offset points are similar with each other between WHH and GL formulars.

According to the Ginzburg-Landau theory, the $H_{c 2}(0)$ is related with the superconducting coherence length $\xi$ given by $H_{c 2}(0)=\Phi_{0} / 2 \pi \xi^{2}$. The coherence lengths at zero temperature also evaluated in Table 1. The coherence length of $\mathrm{NbSe}_{2}$ crystal is about $6.4 \mathrm{~nm}$. Previous reports evaluated the coherence lengths by $\xi_{c}(0)=2.4 \mathrm{~nm}$ and $\xi_{a b}(0)=7.9 \mathrm{~nm}$ along the c-axis and ab-plane, respectively and other reports presented the $\xi=5.8 \mathrm{~nm}^{34,51}$. So, the obtained $\xi=6.4 \mathrm{~nm}$ is not far from the previous reported ones. The coherence lengths of the $\mathrm{NbP} / \mathrm{NbSe}_{2}$ composites are decreased comparing with the pristine $\mathrm{NbSe}_{2}$ crystal such as $3.87 \mathrm{~nm}$ for $\mathrm{NbP} / \mathrm{NbSe}_{2}$ (1:1) and $4.22 \mathrm{~nm}$ for $\mathrm{NbP} / \mathrm{NbSe}_{2}$ (2:1) composites, respectively from the onset points and GL theory reference. On the other hand, the coherence lengths of the $\mathrm{NbP} / \mathrm{NbSe}_{2}$ composites from the offset points are not 
significantly decreased with the pristine $\mathrm{NbSe}_{2}$ such as $8.93 \mathrm{~nm}$ for $\mathrm{NbSe}_{2}, 7.3 \mathrm{~nm}$ for $\mathrm{NbP} / \mathrm{NbSe}_{2}$ (1:1) and $8.09 \mathrm{~nm}$ for $\mathrm{NbP} / \mathrm{NbSe}_{2}$ (2:1) composites, respectively. It is also surprising that the stable bulk superconductivity in the $\mathrm{NbP} / \mathrm{NbSe}_{2}$ composites even though the coherence length is much shorter than the $\mu \mathrm{m}$ scale mean grain size of $\mathrm{NbP}$. Therefore, the length scale of proximity effect may not be related with the coherence length.

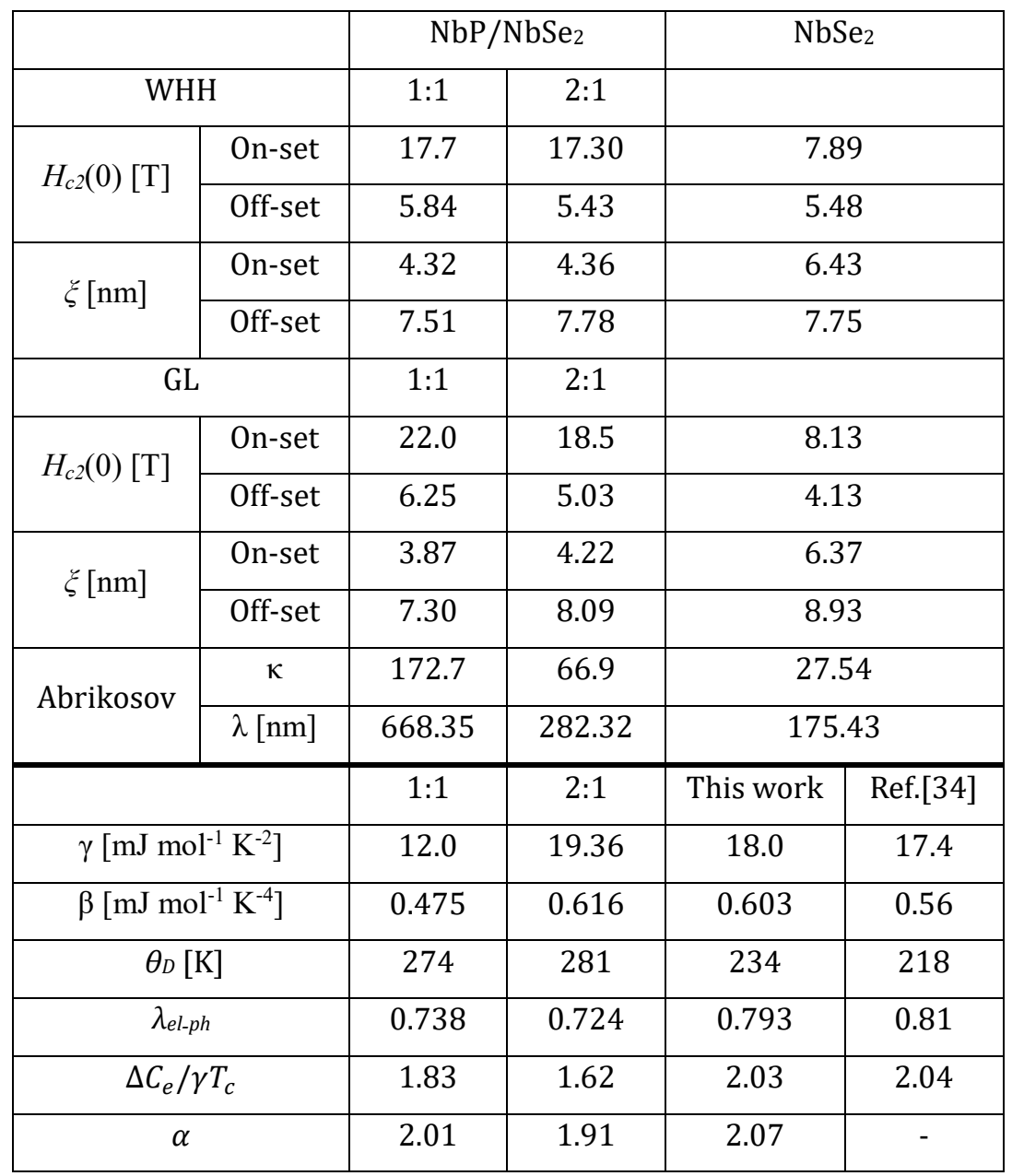

Table 1. Superconducting parameters of the $\mathrm{NbSe}_{2}$ crystal, $\mathrm{NbP} / \mathrm{NbSe}_{2}(1: 1)$, and $\mathrm{NbP} / \mathrm{NbSe}_{2}(2: 1)$ composites. Zero-temperature limit of upper critical field $H_{c 2}(0)$, coherence length $\xi$, Ginzburg-Landau parameter $\kappa$, and penetration depth $\lambda$, obtained by the Werthamer-Helfand-Hohenberg (WHH) formula, Ginzburg-Landau (GL), and Abrikosov theory. Sommerfeld coefficient $\gamma$, phonon coefficient $\beta$, Debye temperature $\theta_{D}$, electron-phonon coupling constant $\lambda_{e l-p h}$, specific heat jump $\Delta C_{e} / \gamma T_{c}$, and superconducting gap parameter $\alpha=\Delta(0) / k_{\mathrm{B}} T_{\mathrm{c}}$ on the $\mathrm{NbSe}_{2}$ crystal and $\mathrm{NbP} / \mathrm{NbSe}_{2}$ composites. 

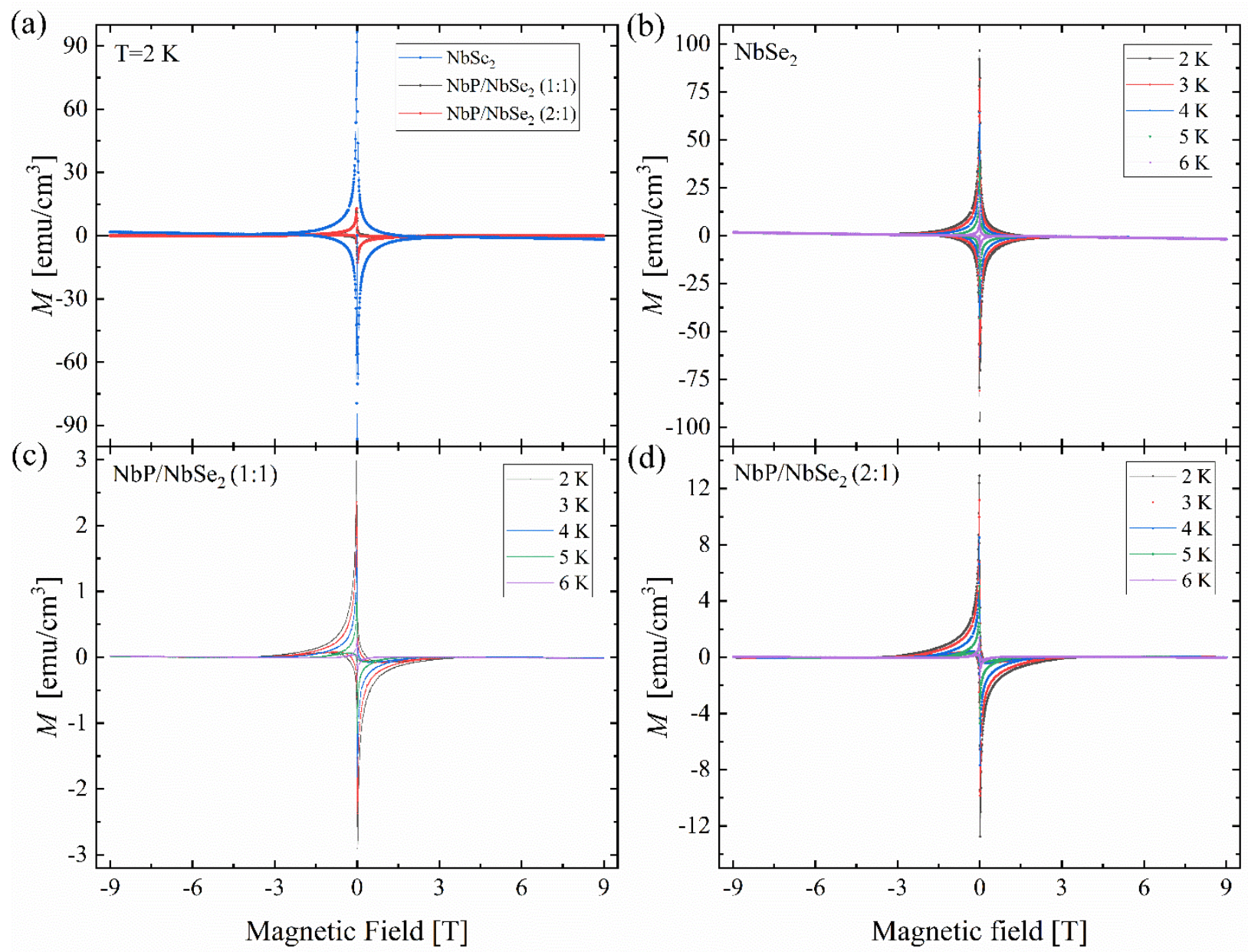

Figure 5. Isothermal magnetic hysteresis of the $\mathrm{NbSe}_{2}$ crystal and composites at $\mathrm{T}=2 \mathrm{~K}(\mathrm{a}), \mathrm{M}(\mathrm{H})$ hysteresis of $\mathrm{NbSe}_{2}$ (b), $\mathrm{NbP} / \mathrm{NbSe}_{2}$ (1:1) (c), and $\mathrm{NbP}_{\mathrm{NbSe}}$ (2:1) (d) composites at different temperatures, as indicated.

In addition, the magnetic-hysteresis loops within an applied magnetic field range of $\pm 9 \mathrm{~T}$ for all the samples at $2 \mathrm{~K}$ is illustrated in Fig. $5 \mathrm{a}$. As expected, the magnetic hysteresis of $\mathrm{NbSe}_{2}$ crystal shows conventional type-II superconducting diamond-like loop. The magnetization of the $\mathrm{NbP} / \mathrm{NbSe}_{2}$ composites are decreased by one order of magnitude or more. Fig. 5b, c, and d present the magnetic hysteresis loop of the $\mathrm{NbSe}_{2}$ and $\mathrm{NbP} / \mathrm{NbSe}_{2}$ composites [(1:1) and (2:1)] at several temperature below $T_{c}$, as indicated.

It is noteworthy that the magnetic hysteresis curves of the $\mathrm{NbP} / \mathrm{NbSe}_{2}$ composites show asymmetric behavior. It implies that the entrance of vortex to the sample during the increase of magnetic field needs more stronger field energy than those of exit of the vortex from the sample during the decrease of magnetic field. The asymmetric $M(H)$ curves in the increasing and decreasing 
field are originated from the bulk pinning and surface barrier effect in a mixed bulk composite ${ }^{52}$. The presence of the non-superconducting NbP phase in the composites would yield complex pinning mechanism in mixed bulk composites at low magnetic fields. When the contribution of the bulk pinning is dominant compared to the surface pinning, the $M(H)$ curves becomes symmetric ${ }^{53}$. It is known that even clean $\mathrm{NbSe}_{2}$ single crystal, there exist significant surface barrier effect 54,55 . On the other hand, this $\mathrm{NbSe}_{2}$ crystal shows stable symmetric $M(H)$ hysteresis loop, indicating the stable bulk pinning effect. In some cases, the significant asymmetric $M(H)$ hysteresis loop has been reported in small doped $\mathrm{NbSe}_{2}$ single crystal56. Therefore, it is not surprising that the significant $M(H)$ hysteresis loop in $\mathrm{NbP} / \mathrm{NbSe}_{2}$ bulk composites.

According to the Abrikosov theory, the $M(T, H)$ is expressed by 57

$$
M(H, T)=\frac{H_{c 2}(T)-H}{4 \pi \beta_{A}\left(2 \kappa^{2}-1\right)}
$$

where $\beta_{A}$ is a constant depending on the vortex arrangement $\left(\beta_{A}=1.16\right.$ for triangular lattice and 1.18 for square vortex), $\kappa=\lambda / \xi$ is the Ginzburg-Landau (GL) parameter, and $\lambda$ is a penetration depth. The field derivative of magnetization near $H_{c 2}$ region can gives rise to the Ginzburg-Landau parameter. The GL parameters and penetration depths are presented in Table 1 from the Abrikosov theory. The penetration depths are calculated using the coherence length obtained by the GL theory. The GL parameter of pristine $\mathrm{NbSe}_{2}$ shows 27.54 which is not significantly different from the ones of previous report. For example, anisotropic GL parameter of $\mathrm{NbSe}_{2}$ crystal reported ${ }^{51} \kappa_{\perp}=11$ and $\kappa_{\|}=$ 29 and $\kappa=33^{34}$. The GL parameters on the composites are significantly enhanced such as 172.5 $\left[\mathrm{NbP} / \mathrm{NbSe}_{2}(1: 1)\right]$ and $66.9\left[\mathrm{NbP} / \mathrm{NbSe}_{2}(2: 1)\right]$. This large values of $\kappa$ are comparable with those of the high temperature superconductors ${ }^{58}$. Because of significant increase of GL parameter in composites, the penetration depths are also significantly increased.

From the magnetic hysteresis measurements, we can obtain the critical current density $J_{c}$ as a function of magnetic field, as presented in Fig. 6a-c. The $J_{c}$ is estimated from the Bean model given by the relation; $J_{c}=20 \Delta M / a\left(1-\frac{a}{3 b}\right)$, where $a$ and $b$ are the width and length of the sample $(a<b)$ and $\Delta M=M_{\uparrow}(H)-M_{\downarrow}(H)^{59}$. The critical current density of $\mathrm{NbSe}_{2}$ crystal reaches up to $1.6 \times 10^{4} \mathrm{~A} / \mathrm{cm}^{2}$, which is one or two order magnitude larger than the previously reported $\mathrm{NbSe}_{2}$ crystal60 $(1.0 \sim 4.0) \times 10^{2} \mathrm{~A} / \mathrm{cm}^{2}$, one the other hand one order of magnitude smaller than the Cr-doped $\mathrm{NbSe}_{2}$ crystal56. $(2.0 \sim 4.0) \times 10^{5} \mathrm{~A} / \mathrm{cm}^{2}$.The critical current density strongly depends on the sample quality and disorder giving rise to strong or weak flux pinning. The $\mathrm{Cr}$-doped $\mathrm{NbSe}_{2}$ crystal exhibited both the strong bulk point flux pinning and surface pinning. The $J_{c}$ values of the composites are decreased 
comparing with the pristine compound such that $500 \mathrm{~A} / \mathrm{cm}^{2}$ and $1900 \mathrm{~A} / \mathrm{cm}^{2}$ for $\mathrm{NbP} / \mathrm{NbSe}_{2}(1: 1)$ and $(2: 1)$ composites, respectively. Even though the $J_{c}$ values are decreased in the composites, it is surprising that the large amount of non-magnetic NbP composite exhibits large critical current density comparable with the previously reported $\mathrm{NbSe}_{2}$ crystal.
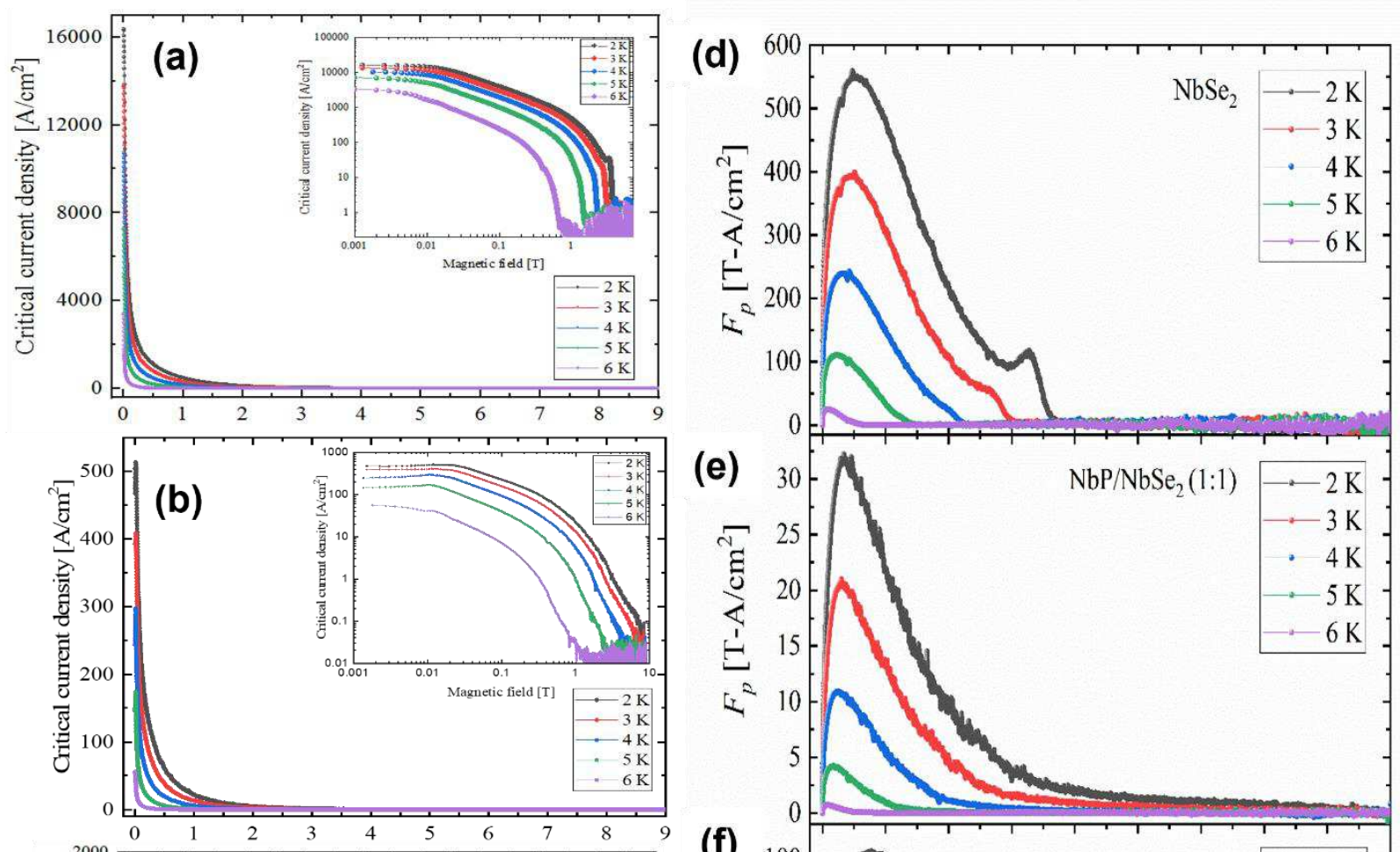

(e)
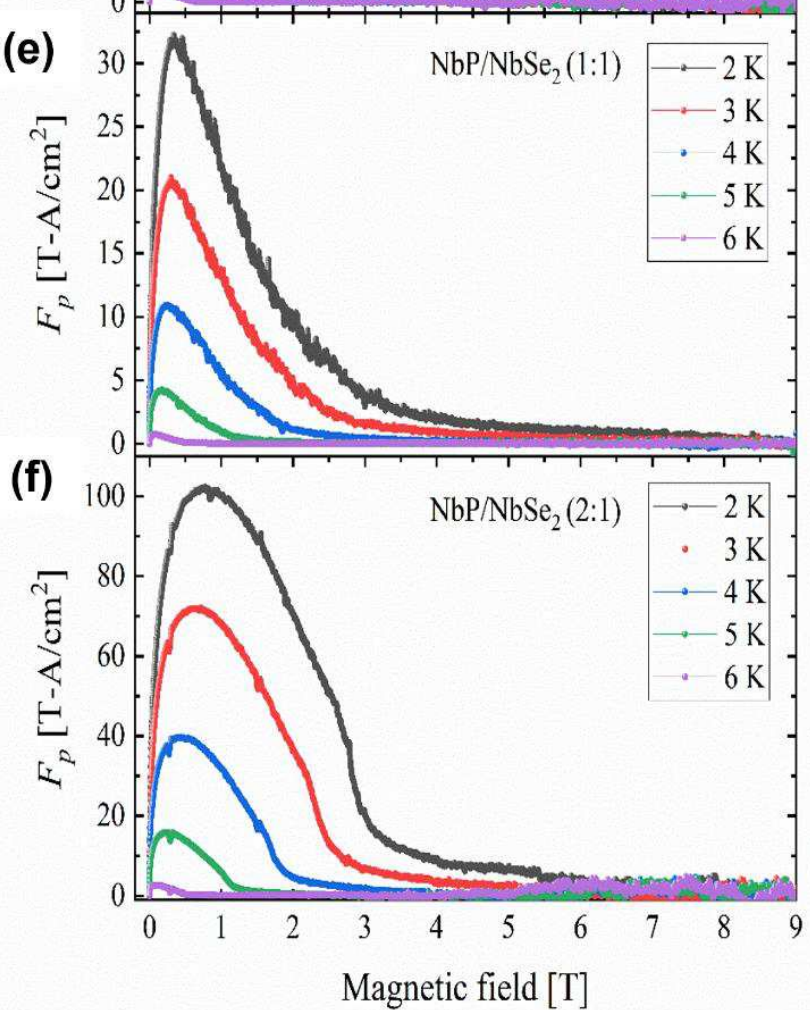

Figure 6. Isothermal critical current density $J_{\boldsymbol{c}}$ and Isothermal pinning force $\boldsymbol{F}_{\boldsymbol{p}}$ with magnetic field $\boldsymbol{H}$ of the $\mathrm{NbSe}_{2}$ crystal (a) and composites of $\mathrm{NbP}_{\mathrm{NbSe}}$ (1:1) (b), and $\mathrm{NbP} / \mathrm{NbSe}_{2}$ (2:1) (c) at various temperature, as indicated. Insets are the log-log scale of the $J_{c}(H)$ curves. Isothermal pinning force $F_{p}$ with magnetic field $H$ of the $\mathrm{NbSe}_{2}$ crystal (d) and composites of $\mathrm{NbP} / \mathrm{NbSe}_{2}(1: 1)$ (e), and $\mathrm{NbP} / \mathrm{NbSe}_{2}(2: 1)$ (f) at various temperature, as indicated. 
When we plot the log-log scale of the critical current density with magnetic field $J_{c}(H)$ as depicted in the insets of Fig. 6a-c, the field dependent behavior shows the saturation at low magnetic fields and rapidly decreasing at high magnetic fields, indicating the formation of vortex bundle and vortex melting at high magnetic fields. It indicates the vortex dynamics on the $\mathrm{NbP} / \mathrm{NbSe}_{2}$ composites is not significantly different with the pristine $\mathrm{NbSe}_{2}$ crystal.

The vortex pinning force is presented in Fig. $6 \mathrm{~d}-\mathrm{f}$, obtained by the $F_{P}=\mu_{0} H \times J_{c}$. The vortex competes with the Lorenz force $F_{L}=I \times H$ and pinning force. When the Lorenz force is balanced with the pinning force, the vortices are pinned in pinning sites. At low magnetic fields, vortices enter in the bulk of the sample and increases the pinning force with increasing magnetic field. For high magnetic fields, vortices become melting state resulting in the weakening of pinning force with increasing magnetic field. The strongest pinning force is observed near 0.5 Tesla for the compounds of $\mathrm{NbSe}_{2}$ and their NbP composites. The pinning forces are decreased with increasing temperatures due to thermal fluctuation. There is a pinning force anomaly near $3.3 \mathrm{~T}$ for $\mathrm{NbSe}_{2}$ at $\mathrm{T}=2 \mathrm{~K}$. That anomalous pinning force region is also observed in the Cr-doped $\mathrm{NbSe}_{2}$ crystal, which is described from the effective disorder of the vortex lattice due to the weak pinning potential called plastic deformation of soft flux line lattice 56,61.

The vortex anomaly is weakened for increasing temperature in $\mathrm{NbSe}_{2}$. While the vortex melting is typical in the $\mathrm{NbP} / \mathrm{NbSe}_{2}$ (1:1) composite for decreasing the pinning force with increasing magnetic field, those of NbP/NbSe 2 (2:1) show an abrupt decrease of pinning force near $3 \mathrm{~T}$ at $2 \mathrm{~K}$. The abrupt change in the field range may be associated with the vortex breakdown due to abrupt vortex melting. It is also noteworthy that strong pinning force in the composite indicates the strong pinning potential in the $\mathrm{NbP} / \mathrm{NbSe}_{2}$ composite in spite of the non-superconducting $\mathrm{NbP}$ composite with large amount of volume fraction.

The superconducting transitions are also observed in specific heat jump near $T_{c}$. Fig.s $7 \mathrm{a} \sim \mathrm{c}$ depict the temperature-dependent specific heat divided by temperature $C_{p} / T$ for $\mathrm{NbSe}_{2}, \mathrm{NbP} / \mathrm{NbSe}_{2}$ (1:1), and $\mathrm{NbP} / \mathrm{NbSe}_{2}$ (2:1) composite samples under static fields as indicated. The specific heat jump near $T_{c}$ on the composites shows bulk superconductivity. To derive the electronic and lattice contributions of the specific heat, we fitted the $C_{p} / T$ as a function of temperature $C_{p} / T=\gamma+\beta T^{2}$ for normal state under high magnetic fields. From the fitting of normal state specific heat (red lines in Fig. 7), we obtain the Sommerfeld coefficient $\gamma$ and phonon coefficient $\beta$, as presented in Table 1. From the Debye model, we can estimate the Debye temperature $\theta_{D}$ by the relation: $\beta=$ $12 \pi^{4} N k_{B} / 5 \theta_{D}^{3}$. The Sommerfeld coefficients of the composites are not changed with the one or pristine compound; $\gamma=18 \mathrm{~mJ} \mathrm{~mol}^{-1} \mathrm{~K}^{-2}\left(\mathrm{NbSe}_{2}\right), 12 \mathrm{~mJ} \mathrm{~mol}^{-1} \mathrm{~K}^{-2}\left[\mathrm{NbP} / \mathrm{NbSe}_{2}(1: 1)\right]$, and $19.4 \mathrm{~mJ} \mathrm{~mol}-1$ 
$\mathrm{K}^{-2}$ [NbP/NbSe 2 (1:1)], implying the electronic density of state is not changed in the composite. On the other hand, the Debye temperatures are little bit increased in the $\mathrm{NbP} / \mathrm{NbSe}_{2}$ composites comparing with the pristine $\mathrm{NbSe}_{2}$ crystal; for example, $274 \mathrm{~K}$ for (1:1) and 281 for (2:1) NbP/NbSe 2 composites while 220 234 K for $\mathrm{NbSe}_{2}$ crystal.
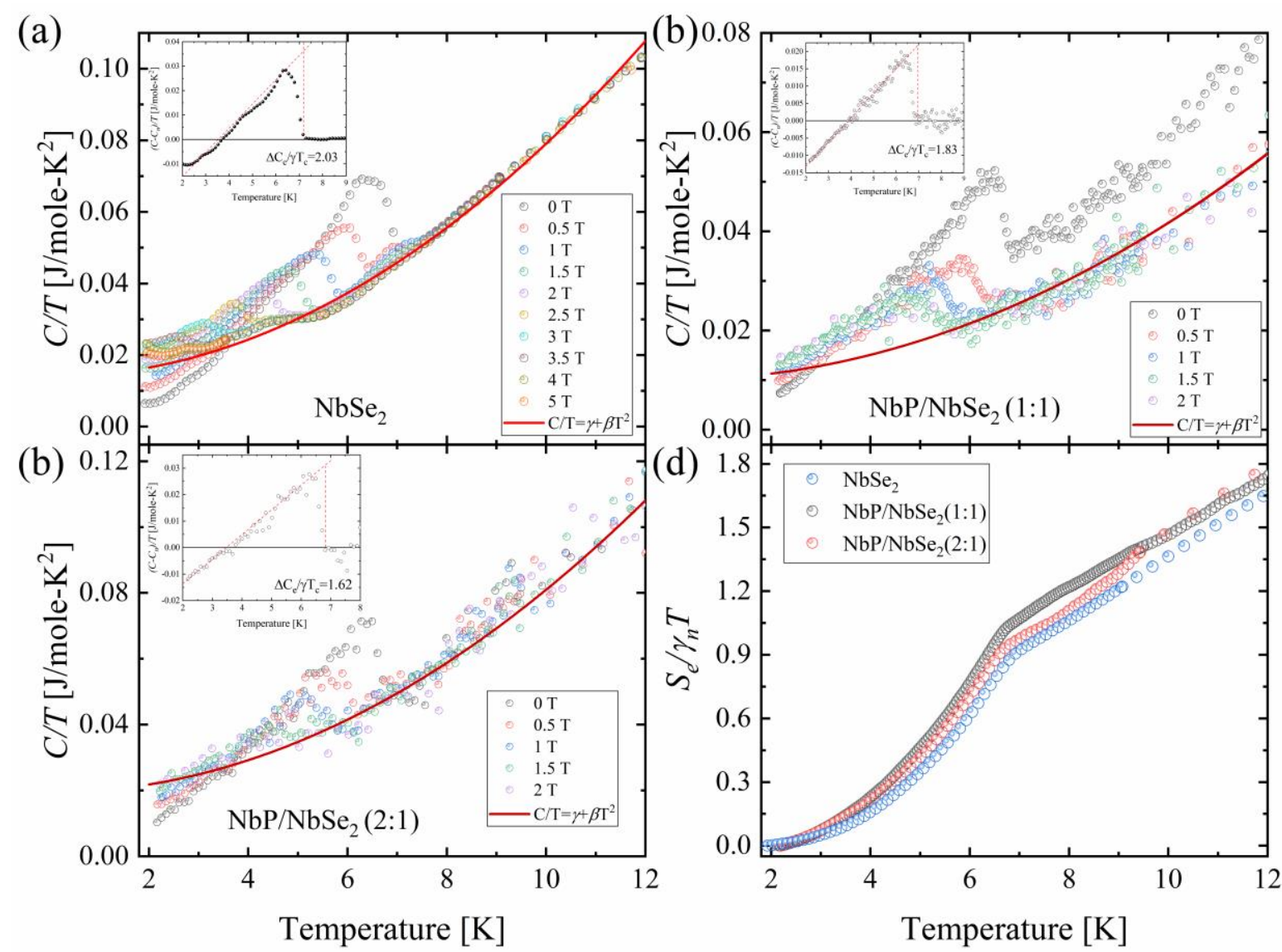

Figure 7. Temperature-dependent specific heat $\boldsymbol{C}_{\boldsymbol{p}}$ divided by temperature $\boldsymbol{C}_{\boldsymbol{p}} / \boldsymbol{T}$ of the $\mathrm{NbSe}_{2}$ crystal (a) and composites of $\mathrm{NbP} / \mathrm{NbSe}_{2}(1: 1)(\mathrm{b})$, and $\mathrm{NbP}_{\mathrm{NbSe}}(2: 1)$ (c) at various MFs (d) Electronic entropy $S_{e}$ scaled by the Sommerfeld coefficient $\gamma_{n}$ and temperature $T\left(S_{e} / \gamma_{n} T\right)$ for $H=0$. Insets of (a) (c) depict the SC specific heat divided by temperature $\left(C-C_{n}\right) / T$ and estimation of specific heat jump (red dotted line).

From the Debye temperature $\theta_{D}$, we can obtain the electron-phonon coupling constant $\lambda_{\text {el-ph, }}$ which is important parameter in Bardeen-Cooper-Schrieffer (BCS) superconductors. The electronphonon coupling constants are calculated to be $0.793,0.738$ and 0.724 using the McMillan formula ${ }^{62}$ : 


$$
\lambda_{e l-p h}=\frac{1.04+\mu^{*} \ln \left(\frac{\theta_{D}}{1.45 T_{c}}\right)}{\left(1-0.62 \mu^{*}\right) \ln \left(\frac{\theta_{D}}{1.45 T_{c}}\right)-1.04}
$$

where $\mu^{*}$ is the pseudo-Coulomb potential and is set to be 0.2 , which is a conventional value in BCS superconductor. The obtained values of $\lambda_{\text {el-ph }}$ of all samples suggest intermediate coupled superconductors ${ }^{57}$ and the coupling constant of composites are comparable or little bit smaller than pristine $\mathrm{NbSe}_{2}$. In spite of non-magnetic NbP composite, it shows the comparable values of electronphonon coupling constant with the pristine $\mathrm{NbSe}_{2}$ crystal.

The insets of Fig.s $7 \mathrm{a} \sim \mathrm{c}$ show the superconducting contribution to the specific heat divided by $T$; $\left(C-C_{n}\right) / T$ by subtracting the normal state specific heat $C_{n}$ from the total specific heat. The plot of $(C$ $\left.C_{n}\right) / T$ versus $T$ shows the lambda-type specific heat jump $\Delta \mathrm{C}_{\mathrm{e}}$ at $T_{c}$. From the BCS theory, the specific heat jump, defined by $\Delta C_{e} / \gamma T_{c}$ is 1.43 for weak coupling limit. We estimated the $\Delta C_{e} / \gamma T_{c}$ values as 2.03 $\left(\mathrm{NbSe}_{2}\right), 1.83$ [NbP/NbSe 2 (1:1)], and 1.62 [NbP/NbSe 2 (2:1)], respectively. The values are higher than those of BCS weak coupling limit (1.43) and are decreased with the increase of NbP concentration in the composites. According to the $\alpha$-model derived from BCS theory, we can roughly estimate the entropy $S$ by subtracting the phonon contribution from the superconducting specific heat and using the following relation 63,64 .

$$
\frac{\Delta C_{e l}}{\gamma_{n} T_{c}}=t \frac{d}{d t}\left(\frac{S}{\gamma_{n} T_{c}}\right)
$$

where $t=T / T_{c}$ is the reduced temperature. The calculated electronic entropy scaled by the Sommerfeld coefficient and temperature $S_{e} / \gamma_{n} T$ is plotted in Fig. $7 \mathrm{~d}$. The electronic entropy is monotonically decreased with decreasing temperature until $T_{c}$ and is exponentially decreased below $T_{c}$. The significant reduction of entropy below $T_{c}$ is consistent with the condensation of superelectrons, which decreases entropy.

From the $\alpha$-model, the superconducting gap can be measured from the relation ${ }^{64}$ :

$$
\frac{\Delta C_{e l}}{\gamma_{n} T_{c}}=1.426\left(\frac{\alpha}{\alpha_{B C S}}\right)^{2}
$$

where the $\alpha$ is the zero-temperature superconducting energy gap scaled by $k_{\mathrm{B}} T_{\mathrm{c}}\left(\Delta(0) / k_{\mathrm{B}} T_{\mathrm{c}}\right)$ and $\alpha_{\mathrm{BCS}}$ $=1.764$. By using the superconducting specific heat jump, the scaled zero-temperature superconducting energy gaps ( $\alpha$-value) are 2.07 for $\mathrm{NbSe}_{2}, 2.01$ [NbP/NbSe 2 (1:1)], and 1.97 $\left[\mathrm{NbP} / \mathrm{NbSe}_{2}(2: 1)\right]$, respectively, which are not sensitive with the $\mathrm{NbP}$ composite concentration and 
are remarkably similar to the reported ones ${ }^{65}$. The gap parameter $\alpha$-value in BCS superconductor is known as $\alpha_{\mathrm{BCS}}=1.764$, so the larger $\alpha$-value in $\mathrm{NbSe}_{2}$ and the composites than $\alpha_{\mathrm{BCS}}$ indicate that the $\mathrm{NbSe}_{2}$ and the related $\mathrm{NbP} / \mathrm{NbSe}_{2}$ composites are not simply described by the BCS superconducting mechanism.

\section{Methods}

\section{Sample preparation}

We separately synthesize the polycrystalline samples of $\mathrm{NbP}$ and $\mathrm{NbSe}_{2}$ from the solid-state reaction using the starting elements of $\mathrm{Nb}$ (99.8 \%, Alpha Aesar, U.S.A.) and P (98.9 \%, Alpha Aesar, U.S.A.) powders and Se (99.999 \%, Alpha Aesar, U.S.A.) granules. The stoichiometric elements of NbP and $\mathrm{NbSe}_{2}$ were sealed in evacuated quartz ampoules, respectively and heat treated at $850{ }^{\circ} \mathrm{C}$ for 48 $\mathrm{h}$ and $850{ }^{\circ} \mathrm{C}$ for $96 \mathrm{~h}$, respectively. The polycrystalline samples of $\mathrm{NbP}$ and $\mathrm{NbSe}_{2}$ were pulverized respectively and mixed with molar ratios of $\mathrm{NbP} / \mathrm{NbSe}_{2}=1: 1$ and $2: 1$ in an agate mortar. The resulting mixed powders were sintered by hot-press sintering at $640{ }^{\circ} \mathrm{C}$, which is much lower temperature of melting point of the compounds to prevent elemental mixing, under a uniaxial pressure of $50 \mathrm{MPa}$ for $1 \mathrm{~h}$.

\section{Characterization}

Structural and phase characterization of the composites were identified by using X-ray diffractometer (Bruker - AXS) with $\mathrm{Cu} \mathrm{K \alpha}$ radiation $(\lambda=0.1546 \mathrm{~nm})$. The grain morphology of the composites was analyzed by the energy-dispersive x-ray spectroscopy (EDX) with high-resolution scanning electron microscopy (HR-SEM, MERLIN, Carl Zeiss). The magnetization was measured by the physical property measurement system (PPMS-14 T Dynacool, Quantum Design, U.S.A.) with vibrating sample magnetometer (VSM). The electrical resistivity was measured by the 4-point probe method using the PPMS-14 T Dynacool system. Specific heat was also measured by the PPMS-14 T Dynacool system using the thermal relaxation method. 


\section{References}

1. Tanaka ,Y., Ren, Z., Sato, T., Nakayama, K., Souma, S., Takahashi, T., Segawa, K. Ando, Y., Experimental realization of a topological crystalline insulator in SnTe, Nat. Phys. 8, 800-803; https://doi.org/10.1038/nphys2442 (2012).

2. Xing, Y., Zhang, L., Wang, J., Topological Anderson insulator phenomena, Phys. Rev. B. 84, 035110;

10.1103/PhysRevB.84.035110 (2011).

3. Li, J., Chu, R.-L., Jain, J. K., Shen, S.-Q., Topological Anderson Insulator, Phys. Rev. Lett. 102, 136806; https://doi.org/10.1103/PhysRevLett.102.136806 (2009).

4. Raghu, S., Qi, X.-L., Honerkamp, C., Zhang, S.-C., Topological Mott Insulators, Phys. Rev. Lett. 100, 156401; 10.1103/PhysRevLett.100.156401 (2008).

5. Nielssen, H., Ninomiya, N., The Adler-Bell-Jackiw anomaly and Weyl fermions in a crystal, Phys. Lett. B. 130, 389-396; https://doi.org/10.1016/0370-2693(83)915290 (1983).

6. Kim, H.-J., Kim, K.-S., Wang, J. F., Sasaki, M., Satoh, N., Ohnishi, A., Kitaura, M., Yang, M., Li, L., Dirac versus Weyl Fermions in Topological Insulators: Adler-Bell-Jackiw Anamaly in Transport Phenomena, Phys. Rev. Lett. 111, 246603; https://doi.org/10.1103/PhysRevLett.111.246603 (2013).

7. Hirschberger, M., Kushwaha, S., Wang, Z., Gibson, Q., Belvin, C. A., Bernevig, B. A., Cava, R. J., Ong, N. P., The Chiral anomaly and thermopower of Weyl fermions in the half Heusler GdPtBi, Nat. Mater. 15, 1161-1165; https://doi.org/10.1038/nmat4684 (2016).

8. Hu, J., Liu, J. Y., Graf, D., Radmanesh, S. M. A., Adams, D. J., Chuang, A., Wang, Y., Chiorescu, I., Wei, J., Spinu, L., Mao, Z. Q., п Berry phase and Zeeman splitting of Weyl semimetal TaP, Sci. Rep. 6, 18674; doi: 10.1038/srep18674. (2016).

9. Kumar, N., Sun, Y., Xu, N., Manna, K., Yao, M., Süss, V., Leermakers, I., Young, O., Förster, T., Schmidt, M., Borrmann, H., Yan, B., Zeitler, U., Shi, M., Felser, C., Shekhar, C., Extremely high magnetoresistance and conductivity in the type -II Weyl semimetals $\mathrm{WP}_{2}$ and $\mathrm{MoP}_{2}$, Nat. Commun. 8, 1642; https://doi.org/10.1038/s41467-017-01758z (2017).

10. Li , Y. et al. Concurrence of superconductivity and structure transition in Weyl semimetal $\mathrm{TaP}$ under pressure, $\mathrm{npj}$ Quantum Mater. 2, 66; https://doi.org/10.1038/s41535-017-0066-z (2017).

11. Wang, W., Kim, S., Liu, M., Cevallos, F. A., Cava, R. J., Ong, N. P., Evidence for an edge supercurrent in the Weyl superconductor MoTe2, Science 368, 534-537; DOI: 10.1126/science.aaw9270 (2020). 
12. Chen, F. C. et al. Superconductivity enhancement in the S-doped Weyl semimetal candidate MoTe2, Appl. Phys. Lett. 108, 162601; https://doi.org/10.1063/1.4947433 (2016).

13. Guguchia, Z. et al. Signatures of the topological $\mathrm{s}^{+-}$superconducting order parameter in the type-II Weyl semimetal $\mathrm{T}_{\mathrm{d}}-\mathrm{MoTe} 2$, Nat. Commun. 8, 1082; https://doi.org/10.1038/s41467-017-01066-6 (2017).

14. Chan, Y. T., Alireza, P. L., Yip, K. Y., Niu, Q., Lai, K. T., Goh, S. K., Nearly isotropic superconductivity in the layered Weyl semimetal WTez at 98.5 kbar, Phys. Rev. B 96, 180504(R); 10.1103/PhysRevB.96.180504 (2017).

15. Wang, R., Hao, L., Wang, B., Ting, C. S., Quantum Anomalies in superconducting Weyl metals, Phys. Rev. B. 93, 184511; 10.1103/PhysRevB.93.184511 (2016).

16. Zhou, T., Gao, Y., Wang, Z. D., Superconductivity in doped inversion-symmetric Weyl semimetals. Phys. Rev. B. 93, 094517; 10.1103/PhysRevB.93.094517 (2016).

17. Hao, L., Ting, C. S., Searching for two-dimensional Weyl superconductors in heterostructures, Phys. Rev. B. 95, 064513; 10.1103/PhysRevB.95.064513 (2017).

18. Khanna, U., Kundu, A., Pradhan, S., Rao, S., Proximity-induced superconductivity in Weyl semimetals, Phys. Rev. B 90, 195430; 10.1103/PhysRevB.90.195430 (2014).

19. Bachmann, M. D. et al. Inducing superconductivity in Weyl semimetal microstructures by selective ion sputtering, Sci. Adv. 3, e1602983; DOI: 10.1126/sciadv.1602983 (2017).

20. Inoue, H., Gyenis, A., Wang, Z., Li, J., Oh, S. W., Jiang, S., Ni, N., Bernevig, B. A., Yazdani, A., Quasiparticle interference of the Fermi arcs and surface-bulk connectivity of a Weyl semimetal, Science 351, 1184-1187; $\quad$ DOI: 10.1126/science.aad8766 (2016).

21. Sergelius, P., Gooth, J., Bäßler, S., Zierold, R., Wiegand, C., Niemann, A., Reith, H., Shekhar, C., Felser, C., Yan, B., Nielsch, K., Berry phase and band structure analysis of the Weyl semimetal NbP, Sci. Rep. 6, 33859; https://doi.org/10.1038/srep33859 (2016).

22. Liu, Z. K., Yang, L. X., Sun, Y., Zhang, T., Peng, H., Yang, H. F., Chen, C., Zhang, Y., Guo, Y. F., Prabhakaran, D., Schmidt, M., Hussain, Z., Mo, S.-K., Felser, C., Yan, B., Chen, Y. L., Evolution of the Fermi surface of Weyl semimetals in the transition metal pnictide family, Nat. Mater. 15, 27-31; https://doi.org/10.1038/nmat4457 (2015).

23. Xu, N., et al. Distinct Evolutions of Weyl Fermion Quasiparticles and Fermi Arcs with Bulk Band Topology in Weyl Semimetals, Phys. Rev. Lett. 118, 106406; https://doi.org/10.1103/PhysRevLett.118.106406 (2017).

24. Shekhar, C., et al. Extremely large magnetoresistance and ultrahigh mobility in the topological Weyl semimetal candidate NbP, Nat. Phys. 11, 645-650; https://doi.org/10.1038/nphys3372 (2015). 
25. Gooth, J., et al. Experimental signatures of the mixed axial-gravitational anomaly in the Weyl semimetal NbP, Nature 547, 324-327; https://doi.org/10.1038/nature23005 (2017).

26. Sun, Y., Wu, S.-C., Yan, B., Topological surface states and Fermi arcs of the noncentrosymmetric Weyl semimetals TaAs, TaP, NbAs and NbP, Phys. Rev. B. 92, 115428; 10.1103/PhysRevB.92.115428 (2015).

27. Huang, S.-M., Xu, S.-Y., Belopolski, I., Lee, C.-C., Chang, G., Wang, B., Alidoust, N., Bian, B., Neupane, M., Zhang, C., Jia, S., Bansil, A., Lin, H., Hasan, Z. M., A Weyl Fermion semimetal with surface Fermi arcs in the transition metal monopnictide TaAs class, Nat. Commun. 6, 7373; https://doi.org/10.1038/ncomms8373 (2015).

28. Niemann, A. C., Gooth, J., Wu, S.-C., Bler, S., Sergelius, P., Hhne, R., Rellinghaus, B., Shekhar, C., Süß, V., Schmidt, M., Chiral magnetoresistance in the Weyl semimetal NbP, Sci. Rep. 7, 43394; https://doi.org/10.1038/srep43394 (2017).

29. Wickramaratne, D., Khmelevskyi, S., Agterberg, D. F., Mazin, I. I., Ising Superconductivity and Magnetism in $\mathrm{NbSe}_{2}$, Phys. Rev. X 10, 041003; https://doi.org/10.1103/PhysRevX.10.041003 (2020).

30. Xing, Y. et al. Ising Superconductivity and Quantum Phase Transition in Macro-Size Monolayer NbSe2, Nano Lett. 17, 6802-6807; https://doi.org/10.1021/acs.nanolett.7b03026 (2017).

31. Saito, Y., Nojima, T., Iwasa, Y., Highly crystalline 2D superconductors, Nat. Rev. Mater. 2, 16094; https://doi.org/10.1038/natrevmats.2016.94 (2017).

32. Xi, X., Wang, Z., Zhao, W., Park, J.-H., Law, K. T., Berger, H., Forró, L., Shan, J., Mak, K. F., Ising Pairing in Superconducting $\mathrm{NbSe}_{2}$ Atomic Layers, Nat. Phys. 12, 139; https://doi.org/10.1038/nphys3538 (2016).

33. Sergio, C., Sinko, M. R., Gopalan, D. P., Sivadas, N., Seyler, K. L., Watanabe, K., Taniguchi, T., Tsen, A. W., Xu, X., Xiao, D., Hunt, B., Tuning Ising Superconductivity with Layer and Spin-Orbit Coupling in Two-Dimensional Transition-Metal Dichalcogenides, Nat. Commun. 9, 1427; https://doi.org/10.1038/s41467-01803888-4 (2018).

34. Luo, H., Nowak, J. S., Li, J., Tao, J., Klimczuk, T., Cava, R. J., S-Shaped Suppression of the superconducting Transition Temperature in $\mathrm{Cu}$-Intercalated $\mathrm{NbSe}_{2}$, Chem. Mater. 29, 3704-3712; https://doi.org/10.1021/acs.chemmater.7b00655 (2017).

35. De Gennes, P. G., Boundary Effects in Superconductors, Rev. Mod. Phys. 36, 225; https://doi.org/10.1103/RevModPhys.36.225 (1964).

36. Meissner, H., Superconductivity of Contacts with Interposed Barriers, Phys. Rev. 117 672; https://doi.org/10.1103/PhysRev.117.672 (1960). 
37. Yoshida, J., Recent Progress of High-Temperature Superconductor Josephson Junction Technology for Digital Circuit Applications, IEICE Trans. Electron. E83-C, 49$59,(2000)$.

38. Ashcroft, N. W., Mermin, N. D., Solid State Physics (Saunders College Publishing, New York, 1976) Ch. 2, p.38

39. Lee C.-C. et al. Fermi surface interconnectivity and topology in Weyl fermion semimetals TaAs, TaP, NbAs, and NbP, Phys. Rev. B 92, 235104; https://doi.org/10.1103/PhysRevB.92.235104 (2015).

40. Bozovic, I., Logvenov, G., Verhoeven, M. A. J., Caputo, P., Goldobin, E., Beasley, M. R., Giant Proximity Effect in Cuprate Superconductors, Phys. Rev. Lett. 93, 157002; 10.1103/PhysRevLett.93.157002 (2004).

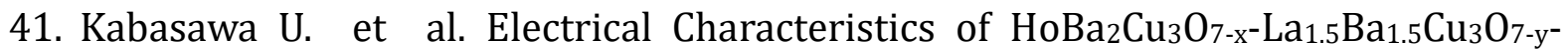
$\mathrm{HoBa}_{2} \mathrm{Cu}_{3} \mathrm{O}_{7-\mathrm{x}}$ Junctions with Planar-Type Structures, Jpn. J. Appl. Phys. 30, 1670, (1991).

42. Yuasa, R., Nemoto, M., Fujiwara, S., Furukawa, H., Mukaida, H., Tokunaga, S., Nakao, M., Long-range Josephson coupling in planar SNS junctions, Physica C (Amsterdam) 185C, 2587; https://doi.org/10.1103/PhysRevB.78.115413 (1991).

43. Barner J. B. et al. All a-axis oriented $\mathrm{YBa}_{2} \mathrm{Cu}_{3} \mathrm{O}_{7-\mathrm{y}}-\mathrm{PrBa}_{2} \mathrm{Cu}_{3} \mathrm{O}_{7-\mathrm{z}}-\mathrm{YBa}_{2} \mathrm{Cu}_{3} \mathrm{O}_{7-\mathrm{y}}$ Josephson devices operating at $80 \mathrm{~K}$, Appl. Phys. Lett. 59, 742; https://doi.org/10.1063/1.105330 (1991).

44. Meltzow A. C. et al. Doped-type coplanar junctions in the $\mathrm{Bi}_{2} \mathrm{Sr}_{2} \mathrm{CaCu}_{2} \mathrm{O}_{8+\delta}$ system, IEEE Trans. Appl. Supercond. 7, 2852; 10.1109/77.621880 (1997).

45. Decca R. S., et al. Anomalous Proximity Effect in Underdoped $\mathrm{YBa}_{2} \mathrm{Cu}_{3} \mathrm{O}_{6+\mathrm{x}}$ Josephson Junctions, Phys. Rev. Lett. 85, 3708; https://doi.org/10.1103/PhysRevLett.85.3708 (2000).

46. Krivoruchko, V. N., Tarenkov, V. Y., Triple -gap superconductivity of $\mathrm{MgB}_{2}-$ (La,Sr)MnO 3 Composite, Phys. Rev. B. 86, 104502; 10.1103/PhysRevB.86.104502 (2012).

47. Zhou, K., Deng, J., Guo, L., Guo, J., Tunable Superconductivity in $2 \mathrm{H}-\mathrm{NbSe} 2$ via In Situ Li Intercalation, Chin. Phys. Lett. 37, 097402; (2020).

48. Werthamer, N. R. , Helfand, E. , Hohenberg, P. C. , Temperature and Purity Dependence of the Superconducting Critical Field, Hc2. III. Electron Spin and Spin-Orbit Effects, Phys. Rev. 147, 295-302; https://doi.org/10.1103/PhysRev.147.295 (1966).

49. Morris, R. C., Coleman, R. V., Bhandari, R., Superconductivity and Magnetoresistance in $\mathrm{NbSe}_{2}$, Phys. Rev. B. 5, 895; https://doi.org/10.1103/PhysRevB.5.895 (1972). 
50. Samuely, P., Szabó, P., Klein, T. , Jansen, A. G. M., Marcus, J., Escribe-Filippini, C., Wyder, P., Upper critical field in $\mathrm{Ba}_{1-\mathrm{x}} \mathrm{K}_{\mathrm{x}} \mathrm{BiO}_{3}$ : Magnetotransport vs. Magnetotunneling, Europhys. Lett. 41, 207-212; (1998).

51. Soto, F., Berger, H., Cabo, L., Carballeira, C., Mosqueira, J., Pavuna, D., Toimil, P., Vidal, F., Electric and magnetic characterization of $\mathrm{NbSe}_{2}$ single crystals: Anisotropic superconducting fluctuations above Tc, Physica C 460-462, 789-790; https://doi.org/10.1016/j.physc.2007.04.032 (2007).

52. Semenov, S. V., Balaev, D. A., Temperature behavior of the magnetoresistance hysteresis in a granular high -temperature superconductor: Magnetic flux compression in the intergrain medium, Physica C 550, 19-26; https://doi.org/10.1016/j.physc.2018.04.005 (2018).

53. Kim, M.-S., Jung, C. U., Park, M.-S., Lee, S. Y., Kim, K. H. P., Kang, W. N., Lee, S.-I., Prominent bulk pinning effect in the $\mathrm{MgB}_{2}$ superconductor, Phy.s. Rev. B 64, 012511; https://doi.org/10.1103/PhysRevB.64.012511 (2001).

54. Bermudez, M. M., Louden, E. R., Eskildsen, M. R., Dewhurst, C. D., Bekeris, V., Pasquini, G., Metastability and hysteretic vortex pinning near the order-disorder transition in $\mathrm{NbSe}_{2}$ : Interplay between plastic and elastic energy barriers, Phys. Rev. B 95, 104505; https://doi.org/10.1103/PhysRevB.95.104505 (2017).

55. Mishra, P. K., Ravikumar, G., Rao, T. V. C., Sahni, V. C., Banerjee, S. S., Ramakrishnan, S., Grover, A. K., Higgins, M. J., Surface barrier effect and the crossover in magnetization relaxation in $2 \mathrm{H}-\mathrm{NbSe}_{2}$, Physica C 340, 64-70; https://doi.org/10.1016/S0921-4534(00)00369-5 (2000).

56. Pervin, R., Krishnan, M., Rana, A. K., Arumugam, S., Shirage, P. M., Effect of Cr atoms in vortex dynamics of $\mathrm{NbSe}_{2}$ superconductor and study of second magnetization peak effect, Mater. Res. Express. 5, 076001; (2018).

57. Doria, M. M., Romaguera, A. R. C., Salem-Sugui, S. Jr., Vanishing of the upper critical field in $\mathrm{Bi}_{2} \mathrm{Sr}_{2} \mathrm{CaCu}_{2} \mathrm{O}_{8+\delta}$ from Landau-Ott scaling, Phys. Rev. B. 76, 052054; https://doi.org/10.1103/PhysRevB.76.052504 (2007).

58. Poole, C. P., Farach, H. A., Creswick, R. J., Superconductivity (Academic Press, New York, 1995, chap. 9, p. 271).

59. Bean, C. P., Magnetization of High-Field Superconductors, Rev. Mod. Phys. 36, 31-39; https://doi.org/10.1103/RevModPhys.36.31 (1964).

60. Spiering, G. A. Revolinsky, E., Beerntsen, D. J., Critical currents in superconducting single crystal Niobium Diselenide, J. Phys. Chem. Solids. 27, 535-541; https://doi.org/10.1016/0022-3697(66)90197-1 (1966).

61. Higgins, M. J., Bhattacharya, S., Varieties of dynamics in a disordered flux-line lattice. Physica C 257, 232-254; https://doi.org/10.1016/0921-4534(95)00704-0 (1996). 
62. McMillan, W. L., Transition temperature of Strong-Coupled Superconductors, Phys. Rev. 167, 331-344; https://doi.org/10.1103/PhysRev.167.331 (1968).

63. Singh, D., Barker, J. A. T., Thamizhavel, A., Hillier, A. D., Paul, McK. D., Singh, R. P., Superconducting properties and $\mu \mathrm{SR}$ study of the noncentrosymmetric superconductor Nb0.5Os0.5, J. Phys. Condens. Matter. 30 075601-075601, (2018).

64. Johnston, D. C., Elaboration of the $\alpha$-model derived from the BCS theory of Superconductivity, Supercond. Sci. Technol. 26, 115011; (2013).

65. Huang, C. L., Lin, J.-Y., Chang, Y. T., Sun, C. P., Shen, H. Y., Chou, C. C., Berger, H., Lee, T. K., Yang, H. D., Experimental evidence for a two-gap structure of superconducting $\mathrm{NbSe}_{2}$ : A specific-heat study in external magnetic fields, Phys. Rev. B. 76, 212504; https://doi.org/10.1103/PhysRevB.76.212504 (2007).

\section{Acknowledgment}

This research was supported by a grant from Kyung Hee University (20171203), Korea and by the National Research Foundation of Korea(NRF) funded by the Ministry of Education, Science and Technology (NRF-2020R1A2C2009353, NRF-2020K1A4A7A02095438). Dr. Omkaram Inturu was supported by the National Research Foundation of Korea(NRF) funded by the Ministry of Education, Science and Technology (NRF-2019H1D3A2A02102233).

\section{Author contribution statement}

Jong-Soo Rhyee: designed and advised the work, analyze the data, revised the manuscript. Yejin Lee: conducted the whole work from experiment, analyze the data, and write a draft. Omkaram Inturu: conducting an experiment and revised the manuscript. Jin Hee Kim: conducted the experimental work.

\section{Additional information}

\section{Declaration of Competing Interest}

The authors declare that they have no known competing financial interests or personal relationships that could have appeared to influence the work reported in this paper. 